\title{
Puffing Frequency and Nicotine Intake in Cigarette Smokers
}

\author{
HEATHER ASHTON,* D.M., M.R.C.P. ; D. W. WATSON, † B.A., M.PHIL. \\ WITH THE TECHNICAL ASSISTANCE OF R. MARSH, $\ddagger$ AND J. SADLERS
}

British Medical fournal, 1970, 3, 679-681

\begin{abstract}
Cummary: The smoking behaviour of 36 subjects smok$S$ ing cigarettes with different filter retention efficiencies for nicotine was studied. Subjects were observed while performing various tasks on a driving simulator and also during a resting period after the tasks. Smokers of cigarettes with high-retention filters took more frequent puffs and obtained nearly the same amount of nicotine as smokers of cigarettes with low-retention filters, both while performing the tasks and during the resting period. Smokers of both types of cigarettes took significantly more puffs and obtained more nicotine per unit time during the resting period than during the tasks. The results are compatible with the possibility that smokers automatically adjust the nicotine dose obtained from a cigarette to some "optimum" level which may vary with different activities.
\end{abstract}

\section{Introduction}

During the course of experiments designed to test the effect of smoking on complex perceptual-motor tasks it became apparent that subiects smoked different types of cigarettes at different rates. Armitage et al. (1968) suggested that some people smoke in order to dose themselves with nicotine and pointed out that a cigarette smoker has "literally finger-tip control of how much nicotine he takes into his mouth." Thus it is possible that smokers may unconsciously adjust their nicotine dose to some desired level, and for this reason it was of interest, as part of the larger study, to investigate the smoking behaviour of subjects given cigarettes with filters of differing retention efficiency for nicotine. To obtain a given quantity of nicotine from a cigarette with a high-retention filter the smoker would need to take more puffs or longer and deeper puffs than if he were smoking a cigarette with a less efficient filter.

\section{Methods}

A total of 36 volunteer subjects were studied, 15 women and 21 men. All were smokers and their normal daily cigarette consumption ranged from 3 to 30 . The age range was from 19 to 35 years. Many of them were university students, but the group also included hospital staff and clerical personnel from an industrial firm.

Cigarettes were kindly supplied by the Tobacco Research Council and were of two types: (a) low-nicotine cigarettes and (b) high-nicotine cigarettes. The filters and blends of tobacco used were such that if the cigarettes were smoked in an analytical smoking-machine with $25-\mathrm{ml}$. puffs of 2 seconds' duration taken once per minute and smoked to a butt length of $20 \mathrm{~mm}$. (the average manner of smoking among a population) (Armitage et al., 1968), the tip retention efficiency for nicotine was $61 \%$ and $22 \%$ and the mainstream smoke contained 1.0 and $2.1 \mathrm{mg}$. nicotine in types $(a)$ and $(b)$ respectively. There was no detectable difference in flavour or other qualities between the two types of cigarettes.

\footnotetext{
Lecturer in Pharmacology.

Honorary Senior Research Associate.

Electronic Technician.

Technician.

Department of Pharmacology, University of Newcastle upon Tyne, and Department of Clinical Fharmacology, Royal Victoria Infirmary,
Newcastle upon Tyne.
}

\section{Experimental Procedure}

The subjects performed tasks on a driving simulator, the details of which will be published later. Briefly, the tasks consisted of "driving" to a film projected on to a screen in front of the subject and responding to certain light signals on a panel below the screen requiring braking, steering, and movements of the trafficator switch. There were 122 such signals in each task. Two 20-minute tasks were used which differed in the degree of "stress" they induced in the subject. Task level 1 involved driving to a film and responding to light signals that corresponded with the requirements of the film. Task level 2 involved driving to the same film but also responding to light signals that were sometimes intentionally contradictory to the driving requirements of the film. Task level 2 was thus the more difficult and stressful because of the uncertainty induced by the conflicting signals. The tasks were performed consecutively in a random order with a resting period of 5 to 10 minutes between each task.

During the first half of each task the subjects smoked one cigarette with no restrictions on the manner or rate of smoking. Nineteen subjects smoked high-nicotine cigarettes and 17 subjects smoked low-nicotine cigarettes. After completion of the two tasks a further half cigarette (approximately) was smoked during a resting period. Ideally a whole cigarette should have been smoked during the resting period. Some of the subjects, however, were not accustomed to smoking as many as three cigarettes in an hour, and in order to obtain a more natural smoking pattern it was decided to limit their nicotine intake where possible. The two groups thus smoked $2 \frac{1}{2}$ cigarettes in a 60-minute period with at least 15 minutes of nonsmoking between the end of one cigarette and the start of the next.

\section{Measurements}

Puff Frequency.-Each puff at a cigarette taken by the subjects during the tasks and resting periods was recorded $b$ : an observer operating a marker on the pen recorder, and the time of starting and finishing each cigarette was also noted. All subiects were told that they were smoking "ordinary" tipped cigareties and none was aware that their puff frequency was being observed.

Analysis of Cigarette Stubs for Nicotine Content.-The cigarette stubs were analysed for nicotine content by $\mathbf{M r}$. C. Grant, Chemistry Department, Tobacco Research Council. Since the tip retention efficiency of the filter and the amount of nicotine retained in the tip were known, it was possible to estimate the amount of nicotine presented to the smoker for each cigarette as follows:

$$
\begin{aligned}
& \mathrm{TR}=\text { tip retention efficiency } \\
& \mathrm{Ns}=\text { nicotine presented to smoker } \\
& \mathrm{Nr}=\text { nicotine retained in tip } \\
& \mathrm{TR}=\frac{\mathrm{Nr}}{\mathrm{Ns}+\mathrm{Nr}} \\
& \mathrm{Ns}(\mathrm{TR})+\mathrm{Nr}(\mathrm{TR})=\mathrm{Nr} \\
& \mathrm{Ns}=\frac{\mathrm{Nr}(1-\mathrm{TR})}{\mathrm{TR}}
\end{aligned}
$$

The nicotine presented to the smoker represented the total amount of nicotine taken into the mouth for each cigarette. 
In the absence of suitable methods for determining blood nicotine levels it was not possible to determine how much of this nicotine was absorbed from the buccal mucosa or from the lungs.

Other Measurements.-Respiratory rate was measured by impedance pneumography by means of two Devices skin electrodes applied to the ninth intercostal space in the midaxillary line connected to an Impedance Rheograph (E. and M. Instrument Company) and recorded on a Beckman Dynograph recorder. An indication of the depth of each respiration was also obtained by observation of the amplitude of the tracing, and the measurement was thus semiquantitative. Subjects' reaction times to the panel light signals during the task, and physiological functions including heart rate, blood pressure, and calf blood flow were also recorded. Details of these measurements will be reported separately.

\section{Results}

\section{Differences Between Groups Smoking Different Cigarettes}

A comparison was made of the smoking behaviour of the subjects smoking the high- and low-nicotine cigarettes.

Puff Frequency.-During both of the driving tasks and during the resting period after the tasks the subjects smoking the low-nicotine cigarettes took more frequent puffs than those smoking the high-nicotine cigarettes. These differences were highly significant. The puff frequencies in the two groups under the various conditions are shown in the Chart and the Table. As would be expected from the increased puff frequency, the average time taken to finish a cigarette was less in the groups smoking the low-nicotine cigarettes. Records of respiration made during the smoking showed no differences in overall rate or in the depth of respiration at or after each puff between the two groups of smokers. Hence these results suggest that the subjects smoking the low-nicotine cigarettes were attempting to compensate for the high filter retention of nicotine by a faster puffing rate. The puff volume appeared to be relatively constant, though small differences may not have been detected.

Nicotine Delivered to Smoker per Cigarette.-During the less exacting level 1 task and during the resting period there was no significant difference in the amount of nicotine delivered to the smoker from the two types of cigarettes. Thus, inder these conditions, increased puffing rate compensated for higher filter retention, so that smokers extracted about the same amount of nicotine from both types of cigarettes. In the level 2 task, however, the smokers of the low-nicotine cigarettes obtained slightly less nicotine than the smokers of the highnicotine cigarettes. It is possible that the motor demands of the more stressful task level limited the rate of smoking, since subjects in the low-nicotine group were commonly observed to cut short an attempt to take a puff when a driving signal appeared on the screen in front of them. These results are also shown in the Chart and the Table.

\section{Differences within the Smoking Groups}

Differences were also observed within the groups of smokers. Both groups had a higher puff rate during the resting period after the tasks than during the tasks themselves. As mentioned before (see Methods), only half a cigarette was smoked during the resting period. Less nicotine is available to the smoker in the first half of a cigarette, since nicotine condenses in the remaining tobacco as a cigarette is. smoked. Therefore puff frequency calculated from the first half of a cigarette might be expected to be greater than that calculated

Mean Puff Rate, Time Taken to Smoke Cigarette, and Nicotine Delivered to Smoker during Task Levels and Resting Period after Tasks for Smokers of Low- and High-nicotine Cigarettes

\begin{tabular}{|c|c|c|c|}
\hline Means & $\begin{array}{l}\text { Low-Nicotine } \\
\text { Cigarettes } \\
\text { (means of } 17 \\
\text { subjects) }\end{array}$ & $\begin{array}{l}\text { High-Nicotine } \\
\text { Cigarettes } \\
\text { (means of } 19 \\
\text { subjects) }\end{array}$ & $\begin{array}{c}\text { Significance Levels } \\
\text { of Differences } \\
\text { Between Low- and } \\
\text { High-Nicotine } \\
\text { Cigarettes }\end{array}$ \\
\hline 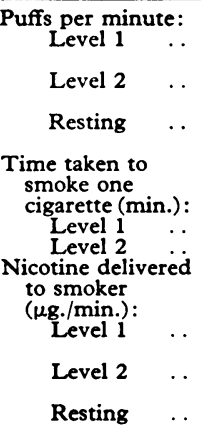 & 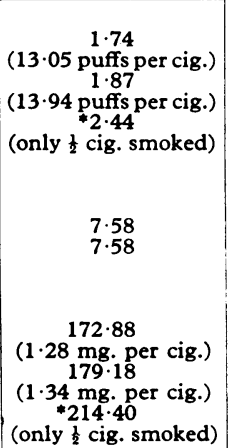 & \begin{tabular}{|c}
0.98 \\
$(9.27$ puffs per cig.) \\
1.02 \\
$(8.89$ puffs per cig.) \\
(only $\frac{1}{2}$ cig. smoked) \\
\\
\\
9.11 \\
8.89
\end{tabular} & $\begin{array}{l}\text { Not significant } \\
\qquad \mathrm{P}<0.05 \\
\text { Not significant }\end{array}$ \\
\hline
\end{tabular}

-Note that puff rate and nicotine delivered were greater $(P<0.01)$ during resting period than during performance of tasks for both low- and high-nicotine cigarettes (see text).
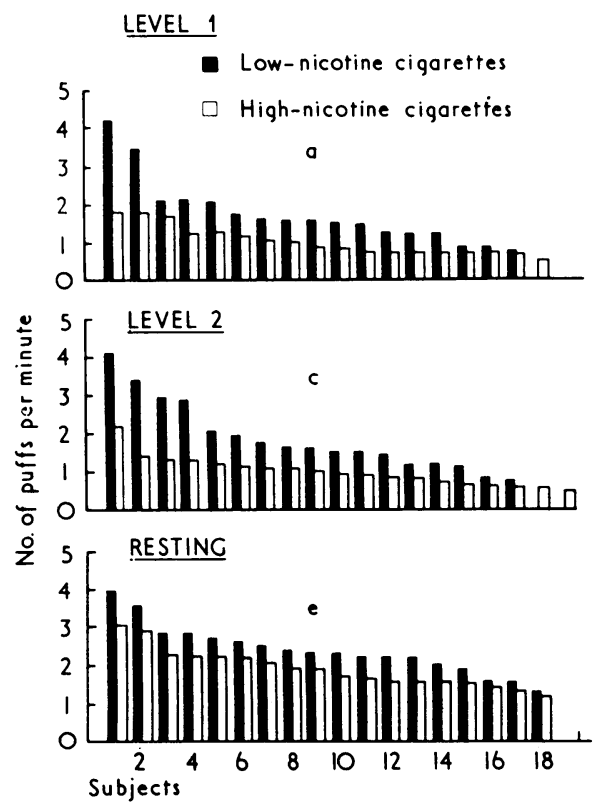

Subjects

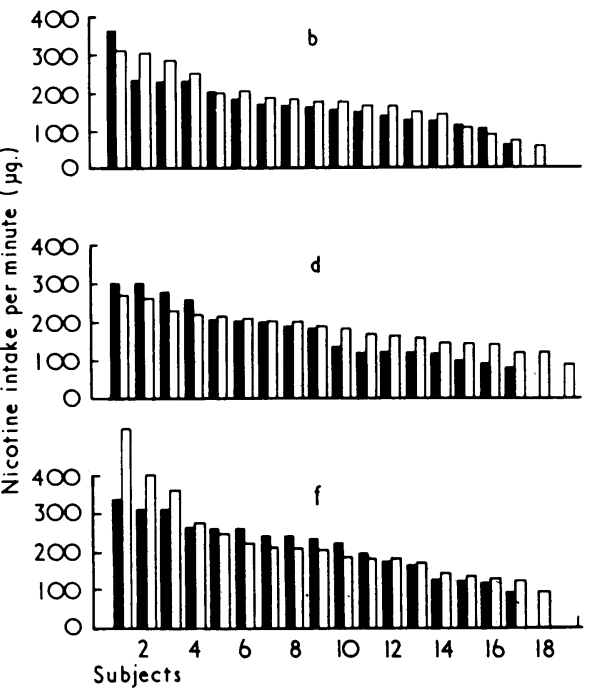

Puff frequency and nicotine presented to smoker in subjects smoking low- and high-nicotine cigarettes during performance of driving tasks levels 1 and 2, and during resting period. Puff frequency is significantly greater in smokers' of low-nicotine cigarettes under all conditions (a, c, and $e$ ). There is no significant difference in nicotine presented to smokers during task level 1 and resting period ( $b$ and $f$ ), but during task level 2 the smokers of low-nicotine cigarettes obtained slightly less nicotine $(P<0.05)(d)$. 
from a whole cigarette. Allowance was made for this factor, however, by comparing the resting puff rate per minute with the puff rate per minute for the first half of the cigarette only during the driving tasks. When compared in this way the resting puff rate was still significantly greater than that during the task levels $(P<0.01)$. In addition, the nicotine obtained from the cigarettes per unit time was also significantly greater than during the tasks in both groups. This finding suggests the possibility that the subjects were striving for a higher nicotine dose during the resting period. There was also a tendency in both the high- and low-nicotine cigarette groups to take more puffs and obtain more nicotine during the level 2 task than during the level 1 task. These differences did not reach statistical significance, but they suggest again that different nicotine doses are "optimum" for different activities. These results are included in the Table.

\section{Discussion}

This investigation shows that under varying conditions smokers of low-nicotine cigarettes had a higher puff frequency and drew into their mouths nearly the same amount of nicotine as smokers of high-nicotine cigarettes. In the absence of suitable methods for determining blood levels of nicotine there is unfortunately no way of measuring how much of the nicotine taken into the mouth was absorbed into the body; but all subjects inhaled when smoking and there was no detectable difference in the mean depth of respiration between the groups smoking different cigarettes. These findings are thus consistent with the possibility that there exists an "optimum" nicotine dose for a given activity and that smokers unconsciously modify their smoking patterns in an attempt to obtain this dose.

This proposition in turn suggests that nicotine exerts in man some central pharmacological action. Subjective evidence from smokers that they are either "tranquillized" or "stimulated" by smoking a cigarette (Armitage et al., 1968) would seem to support this thesis. Several authors have attempted to obtain objective evidence in man. For example, Hauser et al. (1958) and Wechsler (1958) observed changes in E.E.G. patterns in subjects smoking cigarettes, but these changes also occurred to some extent when denicotinized cigarettes were used. Murphree et al. (1967), however, stated that smoking cigarettes or cigars caused changes in E.E.G. patterns, usually of a type associated with stimulation rather than tranquillization. Lambiase and Serra (1957) also concluded that smoking caused short-lived flattening of E.E.G. potentials.

In animals there is now good evidence that nicotine given by intermittent intravenous injection or by cigarette smoke blown into the lungs, in amounts comparable to those obtained by a human subject inhaling a cigarette, causes changes in electrocortical activity and in cortical acetylcholine release (Armitage et al., 1968, 1969; Hall, 1970). Small frequent doses of nicotine tend to cause effects associated with central stimulation (desynchronization of electrocorticogram and increased cortical acetylcholine release), while larger doses given less frequently sometimes cause depressant effects (decrease in cortical activity and cortical acetylcholine output). The results of behavioural studies in rats and mice (Morrison, 1967, 1968a, 1968b) fit in with these observations, since small frequent intravenous doses of nicotine tend to stimulate bar-pressing behaviour and learning rate while less frequent larger doses sometimes have a depressant effect on these activities.

These findings in animals have yet to be demonstrated in man, but it may be that the smokers in the present tests were striving for a nicotine alerting effect while performing the task levels. It is of interest that with both types of cigarettes the puff rate rose significantly during the resting period after the tasks, and the amount of nicotine obtained per unit of time also rose during this period. Under these resting conditions the subjects may have been attempting to obtain the "tranquillizing" effect of higher doses of nicotine. In addition, with both the high- and the low-nicotine cigarettes the subjects appeared to abstract slightly more nicotine during the more stressful level 2 task than during the relatively straightforward level 1 task. These observations fit in with the suggestion of Armitage et al. (1968) that the effects of nicotine in man probably depend critically on the dose and rate of selfadministration by smoking. It would also appear from the present experiments that the rate of self-administration in man is controlled more by the puff rate than by the depth or duration of inhalation of each puff. The question of whether the "optimum" dose of nicotine, and thus the smoking behaviour, alters significantly at different levels of stress and relaxation in conditions where motor activity does not affect puffing rate would require further study in a carefully selected group of subjects.

The differences in the speed and accuracy of performance at the different task levels between the groups of smokers and a comparison with a group of non-smokers will be reported in a separate paper.

The work was supported by a grant from the Tobacco Research Council. We would like to thank Professor J. W. Thompson, department of pharmacology, University of Newcastle upon Tyne, for help and advice at all stages of the investigation. We also thank Mrs. $M$. Russell for help in analysing the records and Mrs. R. Telford for help in the calculations.

Requests for reprints should be sent to Dr. H. Ashton.

\section{REFERENCES}

Armitage, A. K., Hall, G. H., and Morrison, C. F. (1968). Nature, 217, 331. Armitage, A. K., Hall, G. H., and Sellers, C. M. (1969). British fournal of Pharmacology, 35, 152 .

Hall, G. H. (1970). British fournal of Pharmacology, 38271.

Hauser, H., Schwarz, B. E., Roth, G., and Bickford, R. G. (1958). Electroencephalography and Clinical Neurophysiology, 10, 576 P.

Lambiase, M., and Serra, C. (1957). Acta Neurologica, 12, 475.

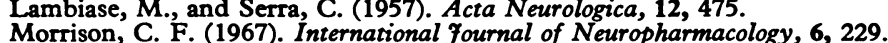
Morrison, C. F. (1967). International fournal of Neurophan

Morrison, C. F. (1968a). Psychopharmacologia, 12, 176. 32, 28 .

Murphree, H. B., Pfeiffer, C. C., and Price, L. M. (1967). Annals of the New York Academy of Sciences, 142, 245.

Wechsler, R. L. (1958). Federation Proceedings, 17, 169 